\title{
Design of a Tunable Broadband Conjugated-Polymer Laser in Solution
}

\author{
Saradh Prasad, K. H. Ibnaouf, M. S. AlSalhi, and V. Masilamani
}

\begin{abstract}
In this work, we had shown that the conjugated-polymer poly(9,9-dioctylfluorenyl-2,7-diyl) (PFO) in benzene can produce amplified spontaneous emission (ASE) with tunablity more than $100 \mathrm{~nm}$ from $410 \mathrm{~nm}$ to $510 \mathrm{~nm}$. And then we had shown that the conjugated polymer poly [2-methoxy-5-(2-ethylhexyloxy)-1, 4-phenylenevinylene] (MEH-PPV) in benzene could produce ASE and lasing from 550 to $650 \mathrm{~nm}$. Finally, we added appropriate molar concentration of these conjugated polymers in benzene to form a solution which has highest optical gain for each conjugated polymer individually. The results showed that these conjugated polymers could lase individually as well as by an energy transfer process from one conjugated polymer to another in tandem.
\end{abstract}

Index Terms-Tunable laser, conjugated polymer, broadband, amplified spontaneous emission (ASE).

\section{INTRODUCTION}

Conjugated polymers have recently attracted the attention of laser scientists because of the rigidity of their molecular structure, which leads to a high fluorescence quantum yield and to a large Stokes shift [1]-[4]. Moreover, the polymers can be easily modified through the attachment of different molecular moieties, which could shift the wavelength region of the lasing action from the UV to the visible to the IR region. These capabilities are comparable to those of conventional laser dyes [5]-[7].

The Stokes shift can be quite large; for example, MEH-PPV, which absorbs at $500 \mathrm{~nm}$ and fluoresces at 560 $\mathrm{nm}$, exhibits a Stokes shift as large as $60 \mathrm{~nm}$. Concentration quenching, which leads to re-absorption, is almost absent. In comparison, the benchmark laser-dye medium R6G has a Stokes shift of only $30 \mathrm{~nm}$ (peak absorption at $520 \mathrm{~nm}$ and emission at $550 \mathrm{~nm}$ ). Finally, conjugated polymers can be easily prepared in the form of neat thin film and optically pumped laser action has been achieved from such thin films [8]-[10]. In due course, the same material has the potential to get electrically pumped to produce laser action. In near future, the conjugated polymers lasers in solid state are a viable reality.

Manuscript received May 12, 2013; revised November 23, 2013. This work was supported by King Saud University (KSU), Deanship of Scientific Research, and College of Science Research Center.

Saradh Prasad is with the Department of Physics, College of science, King Saud University, P.O 2455, Riyadh, 11451, KSA

K. H. Ibnaouf is with Al Imam Mohammad ibn Saud Islamic University, Physics Department, College of Science.

M. S. AlSalhi and V. Masilamani are with King Saud University Riyadh KSA (e-mail: masila123@gmail.com)
In recent years, conducting conjugated polymers have become a promising new gain media for lasers and optical amplifiers [11]-[15]. We have previously reported ASE from the excimeric state of poly [2- methoxy -5- (2- ethylhexyloxy) -1, 4- phenylenevinylene] (MEH-PPV) in liquid solution [16]. Later, in 2013, we also reported a laser from the double excimer of MEH-PPV [17] and poly[9,9-di-(2'-ethylhexyl)fluorenyl-2,7-diyl] (PDHF) [18].

Tunable lasers are needed for research in the medical, engineering and basic science fields. Until recently, few optically pumped tunable lasers, such as Ti: sapphire and first-rate lasers, have been available. Solid-state lasers are very expensive, are delicate to handle and have limited tenability. Dye lasers that use dyes such as Rhoda mine and coumarone derivatives have an excellent capacity for tenability; however, these dyes have shortcomings related to their photochemical stability. In contrast, conjugated polymers are new types of laser materials that exhibit better photochemical stability than conventional dye solutions. Hence, optically pumped, tunable, conjugated-polymer lasers in solution are good competitor to dye lasers in the near future.

\section{EXPEIMENTAL}

\section{A. Materials}

The polymer poly (9, 9-dioctylfluorenyl-2, 7-diyl) (PFO), and poly [2-methoxy-5-(2-ethylhexyloxy)-1, 4-phenylenevinylene] (MEH-PPV) were purchased from American dye source and used as received. The molecular structure is given in Fig. 1(a) and Fig. 1(b).

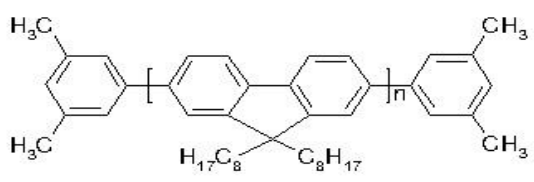

Fig. 1. (a). Molecular structures of polymer of PFO.

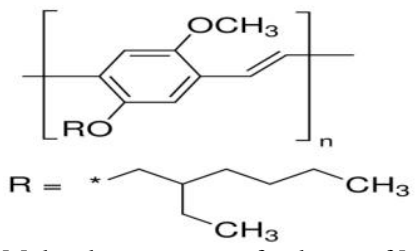

Fig. 1. (b). Molecular structures of polymer of MEH-PPV.

These are macromolecules with a molecular weight of 7264 and (70000 -100000) respectively. The absorption and fluorescence spectra of PFO and MEH-PPV in benzene were studied under wide range of concentrations. 


\section{B. Experimental Setup}

The spectra for the solutions measured using a small quartz cuvette with the dimensions $1 \times 1 \times 4 \mathrm{~cm}$ with an optical path length of $1 \mathrm{~cm}$. UV-VIS absorption spectra were taken using a Perkin Elmer spectrophotometer UV-VIS 950 and the fluorescence spectra were measured on a Perkin Elmer LS55 spectrofluorometer.

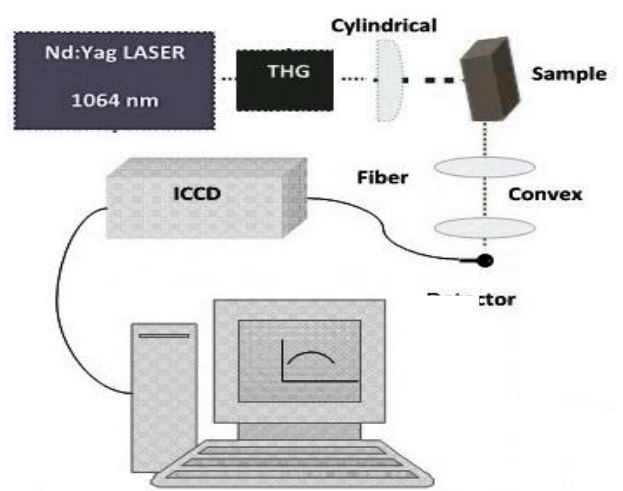

Fig. 2. Schematic representation of the experimental setup.

The main pump source for our work was a frequency-tripled Nd: YAG laser with $5 \mathrm{~ns}$ pulse width. The pump laser was employed to transversely excite the conjugated polymer solution kept in cuvatte. The flat quartz wall of the cuvatte was kept canted to avoid feedback. The output was recorded using an ICCD camera.

\section{RESULTS AND DISCUSSION}

In Fig. 3, spectrum (a) shows the absorption spectra of poly (9, 9-dioctyl fluorenyl-2, 7- diyl) (PFO) in benzene at concentration $22 \mu \mathrm{M}$. It shows that there is an absorption peak at $390 \mathrm{~nm}$ and other absorption peak around $437 \mathrm{~nm}$. Note that the optical density was not to the scale. The peak at 437 $\mathrm{nm}$ could be attributed to ground state aggregation, because this peak was absent for the lower concentrations.

Spectrum (b) of Fig. 3 shows the laser induced fluorescence (LIF) spectrum of PFO in benzene at $22 \mu M$, this is to be compared with absorption spectrum under same concentration. For two absorption peaks, there are three fluorescence peaks. We attribute the LIF peaks at $420 \mathrm{~nm}$, $445 \mathrm{~nm}$ and $470 \mathrm{~nm}$ to the monomeric state, excimeric state and dimeric state of PFO respectively.

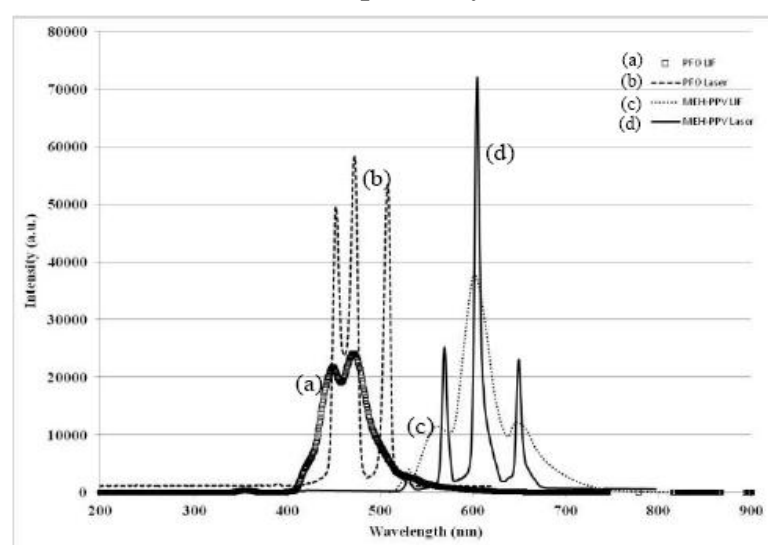

Fig. 3. absorption spectra of (a) PFO, (c) MEH-PPV and LIF of (b) PFO and (d) MEH-PPV

In the same Fig. 3 spectra (c) was absorption of MEH-PPV in benzene as recorded at concentration $100 \mu \mathrm{M}$. It could be found that there were two peaks: one around $333 \mathrm{~nm}$ and the other around $500 \mathrm{~nm}$.

The spectrum (d) was fluorescence spectra of MEH-PPV in benzene at concentration $100 \mu \mathrm{M}$. The result showed that there were three peaks of the LIF: one around $560 \mathrm{~nm}$, next around 600nm and last around 650nm.

\section{A. Tunable Laser}

Fig. 4 shows the spectral profile of LIF and amplified spontaneous emission (ASE) recorded without a cavity resonator. This indicates that the active media in solution have a large gain bandwidth. This ASE bandwidth represents the optical gain bandwidth of any given active medium and this is the measure of range over which laser action is possible, when put into the conventional resonator cavity.

To prepare a proper solution combination, solutions of MEH-PPV at concentration $100 \mu \mathrm{M}$ and PFO at $22 \mu \mathrm{M}$ in benzene were prepared. This two solutions where added in the ratio 1:1. This solution was kept in a cuvette placed in experimental set-up and the output was coupled with ICCD camera to record the spectral results. The pump power of 100 $\mathrm{mJ}$ at $355 \mathrm{~nm}$ was used to excite the sample and the laser spectrum was recorded.

In this mode the ASE output was tunable from 410nm to $470 \mathrm{~nm}$ with average output pulse energy of $10 \mathrm{~mJ}$. At this stage it is important to note that the PFO is capable of producing ASE with three peaks at $425 \mathrm{~nm}, 450 \mathrm{~nm}$ due to the monomer and excimeric state of PFO and there was a peak at $470 \mathrm{~nm}$ which could be due to the dimer with a FWHM of $4 \mathrm{~nm}$. Note the one-to-one correspondence between the LIF shown in Fig. 3 and ASE as shown in Fig. 4.

With the same experimental setup, the output of MEH-PPV was tunable from 550 to $650 \mathrm{~nm}$ with average pulse energy of $30 \mathrm{~mJ}$. It is known from our earlier work that the MEH-PPV produced an ASE at $570 \mathrm{~nm}, 600 \mathrm{~nm}$ and $650 \mathrm{~nm}$ with a FWHM of 8nm [17].

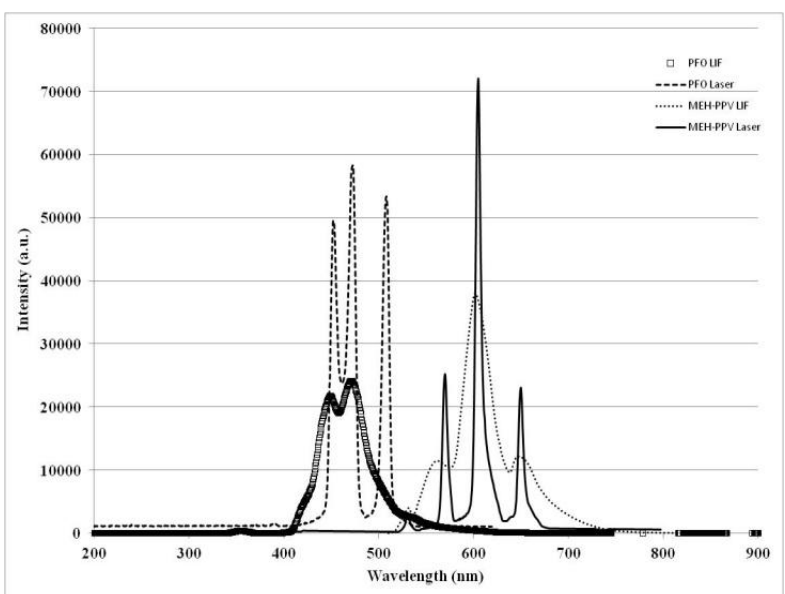

Fig. 4. LIF of (a) PFO, (c) MEH-PPV and ASE of (b) PFO and (d) MEH-PPV.

As shown above the two conjugated polymers produce ASE from $400-500 \mathrm{~nm}$ and then from 550 to $650 \mathrm{~nm}$. In order to fill the gap of $50 \mathrm{~nm}$ (500 to $550 \mathrm{~nm})$, we plan to use one or two of conjugated polymers, such as poly [4, 4'Bis(diphenylvinylenyl)-anthracene] (BDPVA) (emission peak at $509 \mathrm{~nm})$ and poly $\{9,10-\mathrm{Bis}$ 
[(9-ethyl-3-carbazoyl)-vinylenyl]-anthracene $\quad$ (BECVA) (emission peak at $509 \mathrm{~nm}$ ) to get the laser action from 500 to $570 \mathrm{~nm}$. This cocktail solution of benzene, THF is expected to give a flat tunable laser throughout the visible region of the spectrum (from 400 to $650 \mathrm{~nm}$ ).

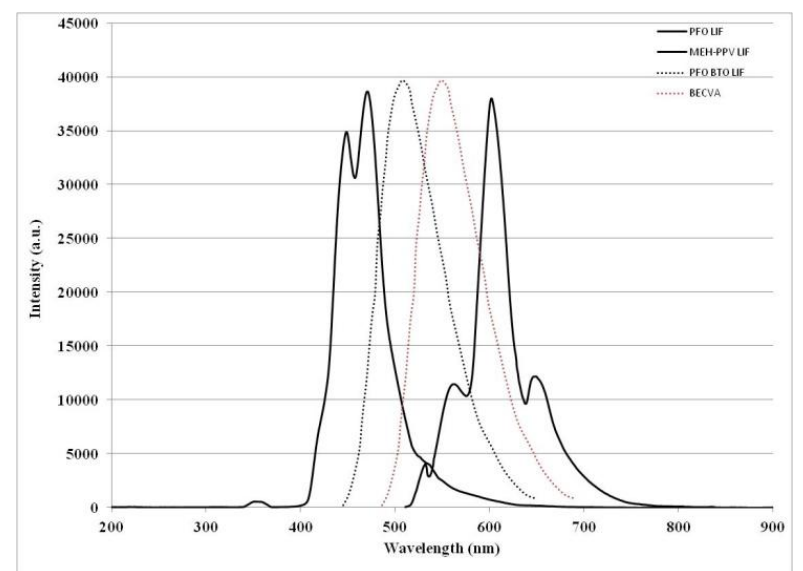

Fig. 5. A projected model of the tuning curves of conjugated polymers in solution.

\section{CONCLUSION}

In this work we had shown that,

The PFO and MEH-PPV mixture solution was capable of high power laser action tunable from 410 to $510 \mathrm{~nm}$ and 550 $\mathrm{nm}$ to $650 \mathrm{~nm}$ respectively.

In near future, a solution mixture which is capable of flat tunable laser from 400 to $700 \mathrm{~nm}$

\section{ACKNOWLEDGMENT}

This project was supported by King Saud University (KSU), Deanship of Scientific Research, College of Science Research Center.

\section{REFERENCES}

[1] J. Heeger, H. Shirakawa, E. J. Louis, S. C. Gau, and A. G. MacDiarmid, "Electrical conductivity in doped polyacetylene," Phys. Rev. Lett., vol. 39, pp. 1098-1101, 1977.

[2] J. H. Burroughes, C. A. Jones, and R. H. Friend, "New semiconductor device physics in polymer diodes and transistors," Nature, vol. 35, pp. 137-141, 1988.

[3] F. Garnier, R. Hajlaoui, A. Yassar, and P. Srivastava, "All-polymer field-effect transistor realized by printing techniques," Science, vol. 265, pp. 1684-1686, 1994

[4] L. Torsi, A. Dodabalapur, L. J. Rothberg, A. W. P. Fung, and H. E. Katz, "Intrinsic Transport properties and performance limits of organic field- effect transistors," Science, vol. 272, no, 5267, pp. 1462-1464, 1996.

[5] A. R. Brown, A. Pomp, C. M. Hart, and D. M. Deleeuw, "Logic gates made from polymer transistors and their use in ring oscillators," Science, vol. 270, no. 5238, pp. 972-974, 1995.

[6] G. Yu, J. Gao, J. C. Hummelen, F. Wudl, and A. J. Heeger, "Advances in synthetic metals: twenty years of progress in science and technology," Science, pp. 1789-179, 1995.

[7] J. J. M. Halls, C. A. Walsh, N. C. Greenham, E. A. Marseglia, R. H. Friend, S. C. Moratti, and A. B. Holmes, "Organic light emitting devices: synthesis, properties and applications," Nature, pp. 498-500, 1995.
[8] G. Kranzelbinder and G. Leising, "Optical Society of Americ,"Rep Prog. Phys, vol. 63, pp. 729, 2000.

[9] M. D. McGehee and A. J. Heeger, "Optical Society of Americ," Adv. Mater, vol. 12, pp. 1655, 2000.

[10] U. Scherf et al., "Curr. Opin. Solid State Mater," Science, pp. 143, 2001.

[11] D. Moses, "Low-loss and highly polarized emission from planar polymer waveguides," Appl. Phys. Lett, vol. 60, pp. 3215, 1992.

[12] G. A. Turnbull, P. Andrew, W. L. Barnes, and I. D. W. Samuel, "Device architecture and materials for organic light-emitting devices," laser Phys. Rev., vol. 64, 2001

[13] C. Bauer, H. Giessen, B. Schnabel, E. B. Kley, C. Schmitt, U. Scherf, and R. F. Mahrt, "Corrugated neat thin-film conjugated polymer distributed feedback laser,” Adv. Mater, vol. 13, pp. 1161, 2001.

[14] W. Holzer, A. Penzkofer, T. Pertsch, N. Danz, A. Bräuer, H. Tillmann, C. Bader, and H.-H. Hörhold, Appl. Phys, vol.74, pp. 333-342, 2002.

[15] J. R. Lawrence, G. A. Turnbull, and I. D. W. Samuel, Appl. Phys. Lett, vol. 80, pp. 3036, 2002.

[16] V. Masilamani, K. H. Ibnaouf, M. S. AlSalhi, and O. A. Yassin, Laser. Phys, vol. 12, pp. 1349, 2007

[17] K. H. Ibnaouf, S. Prasad, M, V. Masilamani, M. S. AlSalhi, A. S Alaamer, J. Europ, "Evidence for the double excimer state of conjugated polymer in a liquid solution," Journal of the European Optical Society, vol. 8, pp. 13001, 2013.

[18] K. H. Ibnaouf, S. Prasad, M, V. Masilamani, and M. S. AlSalhi, "Organic photonic materials and devices XIII," Polymer, vol. 54, pp. 2401-2405, 2013.

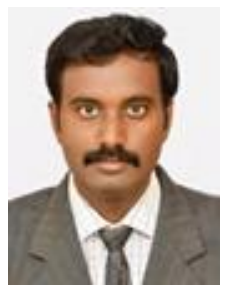

Saradh Prasad was working as a researcher at KSU and the research chair for Laser diagnosis of Cancers since July 2011. His area of specialization is Organic polymer lasers and electronics. He received M. Tech the laser \& electro-optical engineering from college of engineering, Anna University, Chennai.

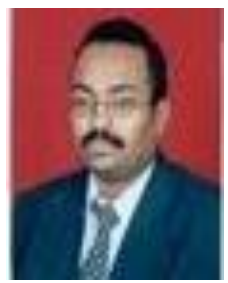

Khalid. H. Ibnaouf was working as assistant professor in Al Imam Mohammad ibn Saud Islamic University, Physics Department, College of Science. Since 2009. His area of specialization is laser physics He received Ph.D. laser physics, physics Department, College of Science, University of AL-Neelain, Khartoum, Sudan (2008)

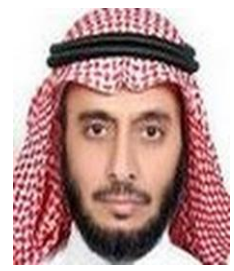

Mohamad. S. AlSalhi holds his Ph.D from University of Hull, England, in laser Physic. He is professor of laser physics and head of research chair in "laser diagnosis of cancer" in King Saud University, Riyadh, KSA. His field of interest is laser spectroscopy, LIBS, laser diagnosis of cancer, Physics of nanotechnology and applications.

He has attended many international and national conferences in laser and nanotechnology topics. He published more than 60 papers in fields of laser and nanotechnology.

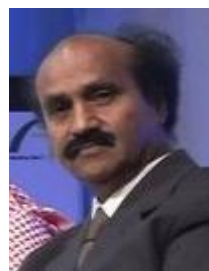

V. Masilamani received his Ph. D from IIT Madras. He had been a visiting scientist in Italy, France, Germany, Canada and USA.

$\mathrm{He}$ is a distinguished professor of laser physics and the research chair professor in "Laser diagnosis of cancer" in King Saud University, Riyadh, KSA.

Author of 65 research papers and 5 US patents. 